\title{
FICHTE Y LA DEDUCCIÓN DEL TIEMPO EN LA WLNM-K (1798)
}

\section{FICHTE AND THE DERIVATION OF TIME IN WLNM-K (1798)}

\author{
ANA CARRASCO-CONDE \\ UCM-UPM CEI Campus de Moncloa*
}

\begin{abstract}
Resumen: Según Fichte, Kant se había limitado a considerar que lo inteligible ordenaba lo sensible, cuando en realidad no lo ordena, sino que lo produce a través de una actividad que no puede ser entendida en ningún caso como creación, sino como un proceder genético-trascendental fundado en el querer. De esta forma el Yo no crea el tiempo, ni se encuentra con él como condición de posibilidad de lo sensible, sino que es deducido en un movimiento generado por la voluntad, esto es, por el querer mismo del Yo. La presente propuesta tratará de analizar, siguiendo la Doctrina de la ciencia nova methodo de 1798, la relación existente entre el querer que se encuentra como fundamento de la actividad del Yo y una deducción del tiempo que trata de apartarse de los planteamientos kantianos.
\end{abstract}

Palabras clave: Fichte, tiempo, deducción, querer, Doctrina de la Ciencia nova methodo.

Aвstract: According to Fichte, Kant confined himself to the belief that the intelligible ordered the tangible, when in truth it does not order it, but rather produces it through an activity that cannot be understood in any case as creation, but as a genetictranscendental course of action based on will. In this way, the I does not create time, nor does it meet with it as a condition of the possibility of the tangible. It is derived in a movement generated by will, that is to say, by the will of the self. This proposal

* Investigadora Postdoctoral Contratada. Dirección postal: Universidad Complutense de Madrid Facultad de Filosofía, Departamento Historia de la Filosofía, Ciudad Universitaria, s/n. 28040 Madrid. E-mail: anacconde@ucm.es.

Research by Ana Carrasco-Conde is supported by a PICATA Postdoctoral contract of the Moncloa Campus of International Excellence (UCM-UPM). 
attempts to analyse the relationship between the will which is the basis of the activity of the I and a derivation of time which tries to deviate from Kantian approaches.

KeYwords: Fichte, time, derivation, will, Wissenschaftslehre nova methodo.

\section{Introducción}

Según Fichte Kant se había limitado a considerar que lo inteligible ordenaba lo sensible, cuando en realidad no lo ordena, sino que lo produce a través de una actividad que no puede ser entendida de ningún modo como creación, sino como un proceder genético-trascendental fundado en el querer. De esta forma el Yo no crea el tiempo, ni se encuentra con él como condición de posibilidad de lo sensible, sino que es deducido en un movimiento de despliegue generado por la voluntad, esto es, por el querer mismo del Yo. Y así, mientras que para Kant el tiempo había de ser entendido como una intuición pura y como forma de la sensibilidad que no procede de lo sensible mismo, sino que constituye la condición trascendental de lo sensible, para Fichte el tiempo mismo es la forma de intuición que unifica lo disperso $(\mathrm{GA} I V / 3,433)^{1}$ pero que es producido por el movimiento de autodeducción y autoconstrucción del Yo. El tiempo surge para nosotros. Por este movimiento el Yo sintetiza los elementos en el tiempo y, al hacerlo, él mismo se sitúa en el tiempo pero, a la vez, como unidad fundante, está fuera del tiempo, en una posición que es «anterior» al tiempo mismo. El Yo es y no es temporal, y como tal, el tiempo que se vivencia sólo puede ser entendido como resultado de la deducción genético/constructiva del propio Yo.

La presente propuesta tratará en primer lugar de analizar esta relación entre el querer que se encuentra como fundamento de la actividad del Yo y una deducción del tiempo que se aleja de los planteamientos kantianos y; en segundo lugar, se esbozará una respuesta a la pregunta, en apariencia obvia, de qué es el «tiempo» para Fichte. Me centraré en un periodo muy concreto de la obra del filósofo: 1798, el ańo de la WLnm (manuscrito de Krause) ${ }^{2}$.

${ }^{1}$ Las referencias pertenecen a la J.G. Fichte. Gesamtausgabe der Bayerische Akademie der Wissenchaften, ed. R. Lauth, H. Jacob y H. Gliwitzky, Stuttgart, Frommann, 1962 y ss. En adelante GA, seguido de serie en números romanos, y tomo y página en arábiga. Cuando haya traducción se indicará tras punto y coma. En concreto esta referencia pertenece a la Doctrina de la ciencia nova methodo que sigue el manuscrito de Krause (WLnm-K).

2 Para este artículo nos centraremos en el manuscrito de Krause, aunque también haremos referencia al manuscrito de Halle. Cuando así se haga será convenientemente indicado con la 


\section{Los «dos» tiempos}

La primera de las claves para entender el concepto del tiempo en Fichte tiene que ver con la diferencia fundamental entre el «yo pienso» kantiano y el "yo actúo» fichteano. Para Fichte el Yo produce, lo que quiere decir que si el principio de todo es el Yo, pero no el "yo pienso", sino un Yo activo y libre que se despliega por la fuerza propulsora de su impulso moral (éste es el sentido del primado de la razón práctica sobre la razón teorética), lo realmente esencial en el Yo no es el ejercicio de la facultad cognoscitiva (como en el Kant de la KrV), sino el despliegue de su conducta práctica. El «yo pienso» kantiano implica que el tiempo no se deduce o, al menos no del modo como lo hará Fichte, sino que se intuye: espacio y tiempo constituyen al sujeto pero no se deducen del sujeto como principio. Les son «dados» (al. gegeben) al Yo y son constitutivos del sujeto al sujetarlo a todo objeto que sale a su encuentro: todo objeto aparece siempre sometido a las condiciones espacio-temporales. Éste es el sentido en Kant de la sensibilidad a priori. No hay un Yo anterior y separado que cree el espacio y el tiempo sino que son maneras, modos de ser, que constituyen al sujeto de afección. El tiempo no procede de lo sensible (lo múltiple, la multiplicidad) o algo de lo que se tenga constancia a partir de la percepción, sino que es condición de posibilidad o, si se quiere, condición trascendental, junto con el espacio, de lo sensible o, dicho de otra forma, la base de todas nuestras intuiciones. Por eso Kant hablará de un carácter ideal del tiempo (y del espacio) aludiendo con ello a los modos generales de la receptividad del sujeto mismo. Como tal el tiempo será entendido como una intuición pura o una forma a priori, trascendental de la sensibilidad ${ }^{3}$. Kant reflexiona sobre este carácter del tiempo en la Estética trascendental, siguiendo los planteamiento de su Dissertatio ${ }^{4}$, para concluir que tanto el tiempo como el espacio sólo pueden ser comprendidos como aquello que posibilita la experiencia y, al mismo tiempo, como aquello que determina las formas del conocimiento. El tiempo por ello constituiría un marco que «se llena»

abreviatura WLnm-H. Existe un excelente comentario del manuscrito de Krause en la Revista Endoxa: "Fichte: la Doctrina de la Ciencia nova methodo», N. 30 (2012), que contiene además un artículo de Vicente Serrano de gran interés para nuestra temática: «La deducción del espacio y el tiempo en los parágrafos 10 a 12 de la Doctrina de la Ciencia nova methodo del manuscrito de Krause».

3 «El tiempo no es otra cosa que la forma del sentido interno, esto es, del intuirnos a nosotros mismos y nuestro estado interno. Pues el tiempo no puede ser una determinación de fenómenos externos. No se refiere a una figura ni a una posición, etc., sino que determina la relación entre las representaciones existentes en nuestro estado interior [...] El tiempo es la condición formal $a$ priori de todos los fenómenos" (KrV B49-B51).

${ }^{4}$ Cfr. Cassirer, 1974, 635ss. 
de realidad ( $\mathrm{KrV}$ B183) al consistir en algo así como el «lugar» en el que la conciencia sitúa a los objetos ${ }^{5}$ mientras que para Fichte, como veremos, el tiempo se deduce a partir de la actividad del Yo como principio de la filosofía: objeto y tiempo (y espacio) se dan a la vez. Así si para Kant el Yo se reduce a ordenar la multiplicidad que se le aparece ( $\mathrm{KrV}$ B50), para Fichte esa multiplicidad sólo puede entenderse a través de la propia actividad del Yo. Toda $m i$ conciencia es un paulatino surgir y construir. Esta diferencia hará a Fichte sostener lo siguiente en la WLnm: "[En Kant] no hay puente entre el mundo sensible y el suprasensible. Pero eso es porque en la Crítica de la razón pura pensó el Yo de forma unilateral y sólo como ordenando lo múltiple, no produciéndolo. La Doctrina de la ciencia tiende fácilmente ese puente. Para ella el mundo inteligible es la condición del mundo de los fenómenos.» (GA IV/3, 424). Ahora bien, ¿en qué consiste este puente o elemento mediador entre lo sensible y lo inteligible? Lo que media es justamente el tiempo. Y así lo afirmará Fichte: «el tiempo es [...] el elemento intermedio entre el mundo sensible y el inteligible.» (GA IV/3, 434) ${ }^{6}$. El tiempo es pues la clave. En el manuscrito de Halle, cuando se aborde la tarea de explicar de forma más precisa este concepto, se encontrará la siguiente afirmación:

Este concepto [de tiempo] es decisivo para el sistema de la Doctrina DE la Ciencia, así como en general para todo el idealismo. Muestra la relación de nuestro sistema con el kantiano y, en particular, con el sistema de la Crítica de la razón pura. De la GÉNesis de este concepto depende sobre todo [este sistema], pues sólo de esta manera se mantiene correctamente el idealismo crítico. La tarea es, por tanto, la siguiente: unificar lo diverso del

\footnotetext{
5 Esta idea llevará a Hegel a entender el tiempo como el «instancia» móvil en el que la conciencia construye sus "figuras" y se reconoce a sí misma: «El tiempo es un absoluto salir-fuera-de$s i$, un engendrarse de lo uno, del punto temporal, del ahora, que de inmediato es el anonadarse de él, y continuamente de nuevo el anonadarse de este mismo perecer; de modo que este engendrarse del no-ser es a la vez simple igualdad e identidad consigo" (Hegel, G.W.F.: Wissenschaft der Logik, Felix Meiner, Leipzig, 1948; trad. de Rodolfo Mondolfo, Ciencia de la Lógica, Ediciones Solar, 5 edic, Buenos Aires, 1982, 244.) Para profundicar en este tema, hay una bibligrafía muy abundante, puede consultarse: Baptista, G. (1988): «Das absolute Wissen. Zeit, Geschichte, Wissenschaft». En: Köhlerm D. - Pöggeler, O. (Eds.): G. W.F. Hegel, Phänomenologie des Geistes, Berlin, Akademie Verlag, 243-259; Bouton, C. (1998): «Éternité et present selon Hegel». En Revue philosophique, 1, 49-70; Murray, M. (1981): «Time in Hegel's Phenomenology of Spirit». En: The review of metaphysics. A philosophicak quaterly 34, n. ${ }^{\circ} 4$, pp. 682-705.

${ }^{6}$ Cfr. Apuntes de Halle. De esta última hay traducción de J. L.Villacańas y M. Ramos en Fichte (1987): Doctrina de la Ciencia nova methodo, Valencia, Natán, p. 113. Esta cita dará título al texto de Peter Rohs «Über die Zeit als das Mittelglied zwischen dem Intelligiblen und dem Sinnlichen». En: Girndt, H.-Schrader, W.H. (Eds.) (1994): Realität und Gewißheit, Fichte-Studien 6, 95-116.
} 
sentimiento [das Mannigfaltige des Gefühls] derivado de una determinación de la voluntad $(\mathrm{GA} \mathrm{IV} / 3,124)^{7}$.

Por lo dicho, el tiempo apuntaría por un lado a algo innegable e irreductible que no tiene que ver con la sensibilidad, pero por otro tiene también una dimensión física. Por eso decíamos que el Yo es y no es temporal porque se sitúa en el tiempo (lo sensible) pero a la vez está fuera de él en una posición anterior al tiempo mismo (lo inteligible). Fichte explicó esta conexión basándose en el concepto de «Querer»: mi querer no es en el tiempo, ya que no está condicionado por nada, pero al mismo tiempo el querer se da en el tiempo: y dado que la actividad del Yo originada por el querer no se pierde en el infinito sino que encuentra siempre un límite esencialmente constitutivo del Yo, el Yo sólo puede percibir su estado paulatinamente y captando cantidades discretas (Quanta) que se dan dentro de una serie ordenada: todo se da de un solo golpe (im einem Schlage) ${ }^{8}$, pero para la exposición filosófica todo queda ordenado temporalmente porque cada elemento es situado uno después del otro: «Las primeras acciones son la base de las siguientes, pero éstas últimas son asimismo condiciones de posibilidad de las primeras, sin las cuales aquéllas se hundirían en una contradicción irresuelta que las haría imposible»? . Por eso frente a la afirmación kantiana de que el tiempo no procede de lo sensible (lo múltiple), Fichte sostiene que el tiempo surge para nosotros en la intuición de una multiplicidad que se deduce y se refiere a la determinación de la voluntad.

Fichte analiza la articulación entre lo sensible (lo múltiple del sentimiento) y lo inteligible (el Yo que quiere) a partir del pensarse a si mismo, como fundamento de la sucesión de lo múltiple ${ }^{10}$. En efecto el Yo al actuar no es sólo libre, sino que también se encuentra limitado: al volver sobre sí a través de sus sucesivas reflexiones para saberse, el Yo analiza su modo de actuar interno y se da cuenta de que, como basamento de todo, hay una unidad que unifica todo lo pensado (lo inteligible) pero también algo que ha de ser unificado (lo sensible) y que limita la actividad del Yo. Y así, al mismo tiempo que el Yo reflexiona sobre sí mismo, sale de sí para darse cuenta de que "algo" lo afecta, algo le ofrece resistencia. Veamos esto más despacio: la actividad del Yo no es inicialmente un actuar, sino un impulso a hacerlo y el querer implica siempre la realización de un esfuerzo

\footnotetext{
Apuntes de Halle. Trad. de José Luis Villacañas y Antonio Ramos, op. cit., p. 110.

${ }^{8}$ Cfr. GA IV/3, 328.

9 Rivera de Rosales, 2012, p. 54.

10 Cfr. GA IV/3, 438.
} 
para conseguir aquello que se quiere. El sentimiento (Gefühl) se manifiesta así como un sentir el límite, como algo sensible que choca contra la esfera inteligible del querer ${ }^{11}$. Aparece así la multiplicidad como un grupo inconexo de elementos diversos sin ninguna relación entre sí, salvo aquella que le ofrezca el Yo. Esta multiplicidad y cómo sea integrada en el querer será lo que «temporalizará» al Yo.

Cuando el Yo sintetiza y determina lo múltiple cae en el tiempo y funda la objetividad ${ }^{12}$. Éste es un tiempo «para nosotros» que alude a la forma de la intuición cuando la voluntad determina su objeto y que surge en la intuición de esa multiplicidad (lo sensible). A este "tiempo" se le opone el concepto de un tiempo «en sí» (inteligible), entendido como entidad independiente, al que ya se ha hecho mención. Podríamos decir para explicar esta síntesis en torno a la voluntad que la primera forma del tiempo («para nosotros») alude a una temporalización del Yo que se ve dentro de una serie que sólo tiene sentido si se aprecia la sucesión que la conforma; y la segunda («en sí») a un tiempo fuera del tiempo que muestra, precisamente el carácter «atemporal» del Yo que se hace a sí mismo: «Me miro a mí mismo en el tiempo, [pero] no estoy en él si me intuyo INTELeCtUAlmente, como determinándome a mí mismo» (GA IV/3, 434). ¿Quiere decir esto que hay dos tiempos en Fichte? ¿Se repite la divergencia existente en Kant entre tiempo y libertad? ${ }^{13}$ La respuesta nos la facilita el mismo Fichte, a la vez que nos proporciona una nueva tarea:

ciertamente los sentimientos aparecen como algo múltiple, DISCRETO, que se da en un tiempo o en otro, en el que el sentimiento y lo sentido deben seguirse. Y entonces surgirían dos tiempos, un tiempo en sí,

11 «El sentimiento es limitación del esfuerzo, por lo tanto el esfuerzo de ir más allá de la esfera del esfuerzo originariamente determinado por el querer puro y a partir de la limitación de ese esfuerzo mediante el querer puro [,] surgiría en el interior de esa esfera el sentimiento de la prohibición más allá de esa esfera del deber». GA IV/3, 439.

12 «El concepto de tiempo depende de ese poner los elementos DISCRETOS unos junto a otros. [Aber] Si juntaran uno y luego de nuevo sólo uno, etc., entonces no surgiría para mi nada diverso, pues para mí no soy uno. Pienso en x,y,z y entonces soy primero [erst $\mathrm{x},[$ dann $] \mathrm{y},[$ dann $]$ z. [Nun] Si x, y, z, tienen [wenig... ein] en común, la conciencia [no] puede hacer una comunidad de $x, y, z . »$ GA IV/3 434.

${ }_{13}$ ¿Eadem, sed aliter? Para Kant la libertad no se da en el tiempo: o es incondicionada e infinita o no es. La libertad sólo es posible allí donde no sucede nada: nichts geschieht (KrV A 541/B 569), donde no hay pasado ni futuro: en lo eterno. Sobre la relación entre tiempo y libertad me permito remitir al lector a A. CARrasco Conde (2009): Chthónos y Dike: la vertebración fichteana en torno a la libertad trascendental kantiana. En: Revista Límite, Tarapacá (Chile), N. ${ }^{\circ}$ 20, pp. 76-97. Sobre la deducción del tiempo en Kant veáse DuQue, F. (2002): La fuerza de la razón. Invitación a la lectura de la Crítica de la razón pura de Kant, Dykinson, Madrid. 
y un tiempo [para nosotros], en los que debería seguirse lo múltiple. Los sentimientos se dan pues an sich [...] en el tiempo y por eso se perciben en el tiempo. Debemos profundizar en este asunto y evitar esos dos tiempos $(\mathrm{GA} \mathrm{IV} / 3,433)^{14}$.

Es preciso por tanto cerrar el hiato o «tender un puente» entre estos dos tiempos, el sensible y el inteligible, precisamente porque el tiempo es el «elemento intermedio» que vincula y vehicula ambos planos. La resolución del problema implica la superación de esta dualidad: no hay dos tiempos, sino una voluntad que se temporaliza y por la cual: 1) Se percibe la sucesión de fases de una actividad en un orden temporal; 2) Ese orden temporal que agrupa la multiplicidad tiene una unidad en su base: el Yo. De esta forma, el Yo se pone como fundamento de lo múltiple (que por sí se presenta discreto y disperso); a la vez que reúne los elementos de la experiencia y los coloca bajo la relación de dependencia, formando una serie de miembros sucesivos. De esta esquematización se deduce el tiempo como forma de la intuición que unifica lo disperso: «el Yo sintetiza los elementos en el tiempo y, por ende, él mismo se vuelve temporal; pero, en cuanto unidad fundante, se sustrae a una posición ontológicamente anterior ${ }^{15}$.

La autodeterminación queda absuelta de la temporalidad y la intuición intelectual permanece ajena al tiempo. Como ser racional finito el hombre está en el tiempo, pero en el fundamento último de todo su pensar se halla esa unidad, concretizada en la intuición intelectual ${ }^{16}$. No hay una experiencia independiente del sujeto que la experimenta, sino que porque el sujeto porta esa unidad puede fundar una experiencia.

Aquí radica otra de las diferencias fundamentales con Kant, puesto que para el de Königsberg la intuición no es intelectual, sino que como «intuición empírica» hace referencia a un objeto dentro del ámbito de lo sensible (intuición empírica) y se da siempre en el tiempo (y en el espacio) entendido éstos como formas a priori de toda percepción posible desde el punto de vista de la sensibilidad. De forma que, si el Yo se intuye a sí mismo como objeto lo haría siempre en el espacio y en el tiempo.

${ }^{14}$ Cfr. GA IV/3, 124; trad. de J.L.Villacańas y M. Ramos en Fichte: Doctrina de la Ciencia nova methodo, op. cit., p. 110.

15 Cfr. Gaudio, 2012, 273-297.

16 Cfr. Tilliette, X. (1995): Recherches sur l'intuition intellectuelle de Kant à Hegel, Vrin, Paris. 
Hay otro tipo de «intuición», la «intuición pura» pero ésta, lejos de estar inscrita en la experiencia, mienta la determinación necesaria para que "haya» algo así como «realidad». Ambas se refieren a un Yo entendido desde su facultad cognoscitiva, no productiva. Y el Yo es ante todo y sobre todo, dice Fichte, actividad, pero no una actividad cualquiera sino la actividad que regresa sobre sí y mantiene su independencia frente a lo empírico. En este sentido Fichte puede decir que no puede afirmarse, como lo hace Kant, que "Todo pensamiento se da en el tiempo» ${ }^{17}$ porque es la intuición intelectual la que deviene sensible en el tiempo y como medio entre lo sensible y lo inteligible. De este modo, frente a Kant, Fichte distingue tres tipos de intuición: la sensible en el espacio; la inteligible de nuestro querer; la de nuestro querer en el tiempo que reúne las dos primeras. Y así, aunque parece que coexisten dos tiempos: el en-sí, asociado a lo inteligible, y el "para nosotros», asociado a lo sensible, hay solo uno. Por eso:

La Doctrina de la ciencia tiende fácilmente ese puente. Para ella el mundo inteligible es la condición del mundo de los fenómenos. Este último se construye a partir del primero. El primero consiste en su verdadero punto central, en el Yo, que sólo es completo en el querer. Todas las representaciones surgen a partir del pensamiento del querer (GA IV/3, 424).

La deducción del tiempo (y del espacio) explicita el elemento central de la Doctrina de la Ciencia que hace explicable toda conciencia y toda experiencia: el querer, como principio de la temporalidad y la espacialidad de la conciencia.

\section{La unidad del tiempo, el tiempo como unidad}

La voluntad pura no puede explicar por sí sola la conciencia real y concreta si no se extiende en el tiempo, si no se hace objeto y deviene causalidad real sobre el mundo sensible. Sólo así es efectiva ${ }^{18}$. Si actuamos en sí no somos en el tiempo,

17 «Todo pensamiento se da en el tiempo, en todo pensamiento se da continuidad a la intuición [des Willens]. Esa expresión no es adecuada, pero es así: en la medida en que refiero la intuición a la multiplicidad [Mannigfaltige] del querer, en esa medida dura [en el tiempo]. Sólo en ese sentido puede el tiempo llamarse forma de la intuición. Es forma de la intuición intelectual, pero en la medida en que adopta esa forma se hace sensible». En GA IV/3, 434.

${ }^{18}$ Cfr. GA IV/3, 448. 
porque la voluntad no es múltiple ${ }^{19}$. El actuar de la actividad del Yo es un actuar en el mundo que, gracias al sentimiento, se me presenta como múltiple y que, por ello, secuencia la causalidad de mi voluntad: antes de abrir una puerta, por ejemplo, he de pasar por el pasillo y sólo después podré entrar en la habitación. Hay por tanto una transición por la que ha de pasar el Yo para realizar un fin determinado:

Ese transitar está condicionado por la CAUSALIDAD de mi voluntad. Cada miembro en la serie es considerado como condicionado por la CAUSALIDAD de mi voluntad. Extiendo mi voluntad en el tiempo, y con ello se extiende también mi voluntad, que actúa a la vez a intervalos. Hace una cosa tras otra, atraviesa estados intermedios y mediante eso es algo limitado en la intuición. Algo que está bajo leyes, que no depende de mí, aparece para mí como dependiente de la fuerza de la naturaleza. Mi efectividad se extiende sólo mediante una resistencia en la naturaleza a través del tiempo (GA IV/3, 429)

Kant diría que siento que (me) pasan cosas, pero el tiempo en sí mismo no pasa, sino que permanece idéntico a sí mismo: un año, por ejemplo, es igual a otro: si los distinguimos es por lo que en ellos (nos) ha pasado ${ }^{20}$. Para Fichte siento que pasa el tiempo porque se suceden mis acciones y sólo así siento que (me) pasan cosas. El tiempo aparece siempre como sucesión de momentos ${ }^{21}$ que hacen ver que aquello que aparecía como disperso e inconexo tiene un eje vertebral que los agrupa en una línea del tiempo que es una línea del tender hacia un fin. Ésta será según Fichte la tarea: «la tarea que nos surge para la solución del tiempo es ésta: la de reunir lo múltiple del sentimiento. Esa reunión se da de modo tal que lo múltiple ha de ser deducido de la determinación de la voluntad y relacionarse con ella.» (GA IV/3, 433)

19 Cfr. GA IV/3, 432.

${ }^{20}$ Cf. KrV A 144 / B 183: «No es el tiempo el que pasa, sino que es la existencia de lo transitorio lo que pasa en él. Al tiempo, que es, por su parte, permanente y no transitorio, le corresponde pues, en el fenómeno lo que posee una existencia no transitoria, es decir, la sustancia. Sólo desde ésta podemos determinar temporalmente la sucesión y la simultaneidad de los fenómenos».

21 «Eso SUCESIVO se da en el tiempo, y cada nuevo poner de mí mismo ocurre siempre en el tiempo, lo SUCESIVO y el tiempo donde se da, son puestos conjuntamente [werden zusammengefasst], y sólo lo formal, no lo material, es captado y gracias a eso es posible pensar un tiempo. Tiempo y momento se comportan como lo determinable frente a lo determinado». GA IV/3, 432 . 


\section{Conclusiones}

El tiempo es pues síntesis, unidad ${ }^{22}$, y su concepto depende de poner juntos todos los elementos que aparecían separados ${ }^{23}$, pero en torno a una voluntad de la que son consecuencia y por la cual hay una sucesión temporal de momentos de una acción. Al mismo tiempo dicha serie sensible implica una vertebración interna que apunta a la unidad inteligible dada por el querer, que media por ello, como puente, entre ambos mundos ${ }^{24}$ : «Situar lo inteligible en lo sensible en cuanto fundamento de la reunión [de ambos] significa: lo meramente pensado no está en la experiencia, sino que es llevado allí mediante el que experimenta» ${ }^{25}$.

El tiempo es de este modo no la instancia inmóvil a introducir, junto con el espacio, sino la red progresiva de reunión en la que quedan enhebrados los diferentes acontecimientos (o los actos que conducen a un determinado fin) dentro de una secuencia causal ordenada de momentos bajo la unidad de la actividad determinada de la voluntad del Yo, una red que va siendo tejida por la propia actividad del Yo.

\section{Bibliografía}

\section{Fuentes}

Fichte, J.G. (1962 y ss): Gesamtausgabe der Bayerische Akademie der Wissenchaften, ed. R. Lauth, H. Jacob y H. Gliwitzky, Stuttgart, Frommann.

- (1978): Doctrina de la Ciencia nova methodo, Valencia, Natán, 1987. Traducción de José Luis Villacañas y Manuel Ramos.

\section{Literatura secundaria}

Bertinetto, A. (Ed.) (2009): Leggere Fichte, Istituto Italiano per gli Studi Filosofici, Fichtiana, Nuova serie 27, Napoles.

${ }^{22}$ Sobre el concepto de unidad Cf. Zöller, G.: «Die Einheit von Intelligenz und Willen in der WLnm». En: Girndt, H.- Navarro Pérez, J.: Zur Einheit der Lehre Fichtes. Die Zeit der Wissenschaftslehre nova methodo, Fichte-Studien, 16 (1999), 91-114.

23 «El concepto de tiempo depende de ese poner los elementos DISCRETOS unos junto a otros». En GA IV/3, 434.

${ }^{24}$ Cf. Rohs, P.: «Über die Zeit als das Mittelglieg zwischen dem Intelligiblen und dem Sinnlichen", op. cit.

${ }^{25}$ GA IV/3, 435. 
Cassirer, E. (1974): El problema del conocimiento, II, Fondo de Cultura Económica, México.

Gaudio, M. (2012): «Lo inteligible y lo sensible. Consideraciones sobre el $₫ 13$ de la Doctrina de la Ciencia nova methodo». En Revista Endoxa. Series filosóficas. La Doctrina de la Ciencia nova methodo de Fichte (1796-1799) en Endoxa. Series Filo-

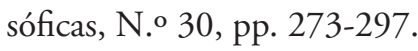

Grüneber, P. (2009): «Wie kann die transzendentale Apperzeption > gehaltvoll< werden bzw. die Rezeptivität spontan?». En: Fichte-Studien 33, pp. 49-63.

Metz, W. (1994): «Fichtes genetische Deduktion von Raum und Zeit in Differenz zu Kant». En Girndt, H.-Schrader, W.H. (Eds.): Realität und Gewißheit, FichteStudien 6.

Oesterreich, P.L.-Traub, H. (2006): Der ganze Fichte. Die populäre, wissenchaftliche und metaphilosophische Erschließund der Welt, Stuttgart, Kohlhammer.

Rivera de Rosales, J. (2012): «Los tres primeros actos del Yo: la intuición intelectual, el concepto del Yo». En Revista Endoxa. Series filosóficas. La Doctrina de la Ciencia nova methodo de Fichte (1796-1799) en Endoxa. Series Filosóficas, N.o 30.

Rohs, P. (1994): «Über die Zeit als das Mittelglied zwischen dem Intelligiblen und dem Sinnlichen». En: Fichte-Studien 6, 95-116.

Schnell, A. (2010): "La déduction du temps chez Fichte et Schelling». En: GallandSzymkowiak, M.-Chédin, M.- Bastian Weiss, M. (Ed.): Fichte - Schelling. Lectures croisées / Grekreuzte Lektüren. Würzburg, Ergon, pp. 107-118.

Serrano Marín, V. (2012): «La deducción del espacio y el tiempo en los parágrafos 10 a 12 de la Doctrina de la Ciencia nova methodo del manuscrito de Krause». En: Rivera de Rosales, J. - Cubo, O. (2012): La Doctrina de la Ciencia nova methodo de Fichte (1796-1799) en Endoxa. Series Filosóficas, N. o 30, pp. 255-272.

Tilliette, X. (1995): Recherches sur l'intuition intellectuelle de Kant à Hegel, Vrin, Paris.

Zöller, G. (1998): Fichte's Transcendental Philosophy. The Original Duplicity of Intelligence and Will, Cambridge University Press, Cambrigde.

- (1999): «Die Einheit von Intelligenz und Willen in der WLnm». En: Fichte-Studien $16,91-114$.

Recibido: 07/11/2012

Aceptado: 24/02/2014 
